\title{
CLINICAL, HORMONAL, AND ULTRASOUND CHARACTERISTICS OF PATIENTS WITH NEWLY DIAGNOSED GRAVES' DISEASE AND DIFFERENT THYROID ANTIBODY PROFILES
}

\author{
R. Mekova, M. Boyanov \\ Clinic of Endocrinology and Metabolism, University Hospital Alexandrovska; Department of Internal Medicine, \\ Faculty of Medicine, Medical University Sofia - Sofia, Bulgaria
}

\begin{abstract}
Objective: Graves' disease (GD) is characterized by elevated TSH-receptor antibodies (TRAb) and less often - thyroid peroxidase (TPOAb) and thyroglobulin antibodies (TgAb). Our aim was to examine the hormonal and ultrasound characteristics of patients with newly diagnosed GD with differing positive thyroid antibodies. Materials and Methods: This study included 249 patients with newly diagnosed GD (191 women, 58 men). $40.2 \%$ of them had Graves' ophtalmopathy. The serum levels of TSH, free T4, free T3, TRAb, TPOAb, and TgAb were measured with third generation ECLIA assays (Roche Diagnostics, Switzerland). Thyroid ultrasound was performed with a Fukuda-Denshi 550 device (Fukuda Corp., Japan) and an Ultrasonix device (Ultrasonix Medical Corp., Canada). Statistical analyses were done using the SPSS 23.0 statistical package (Chicago, IL). Results: $64 \%$ of the patients were TPOAb+ and $36 \%-T g A b+$. One third were only TRAb+, $1 / 3$ had two positive antibodies (TRAb + second antibody) and 1/3 - all three positive antibodies. Patients with more positive antibodies tended to be younger, had higher fT4, TRAb levels, thyroid volume but rarely had nodules on US and accompanying GO. Positive TPOAb antibodies were found in younger patients, with higher fT4 and TRAb levels, higher thyroid volume and lower prevalence of nodules and GO. The same trends were found in patients with positive TgAb. Conclusion: The different characteristics of GD patients with varying thyroid antibody profiles may be due to a variation in the pathogenesis of the disease. An individualized clinical approach may be suitable in those cases.
\end{abstract}

Key words: Graves' disease, Graves' orbitopathy, thyroid antibodies, clinical/hormonal/ultrasound characteristics

Corresponding author: Prof. Mihail Boyanov, Clinic of Endocrinology and Metabolism, University Hospital Alexandrovska; 1, G. Sofiyski str., Sofia 1431, Bulgaria, Fax: +359 29230779 , e-mail: mihailboyanov@yahoo.com

\section{INTRODUCTION}

raves' disease (GD) is the most common cause of thyrotoxicosis due to autoimmune thyroid disease (AITD) [1]. The main driving force of the hyperthyroidism seems to be the presence of TSH-receptor antibodies (TRAb) [2]. High levels of TRAb in hyperthyroidism are one of the cornerstones in the diagnosis of GD, together with increased uniform radio-iodine uptake and diffuse ultrasoud changes [1, 3]. In addition, AITD is marked also by elevation of anti-thyroid peroxidase 
and anti-thyroglobulin antibodies (TPOAb, TgAb) [4-6]. Their positivity and significance are being better studied in the setting of autoimmune thyroiditis. However, they are often elevated in GD. Different studies reported a $50-70 \%$ prevalence of positive TPOAb and $30-50 \%$ positive TgAb in newly discovered GD [7-15]. Their clinical significance is less well understood. Some older studies have linked TPOAb positivity in GD with lower rate of accompanying Graves' ophtalmopathy (GO), higher thyroid volume as well as with lower rates of complete remission of the AITD $[8,9,12,16,17]$. Other studies found an association of anti-TPO positivity with GO in adolescents [18]. In the RISG study the thyroid volume was positively related to the anti-TPO antibodies [16]. Measurement of TPOAb levels 6 months after the initial diagnosis was found useful in the prediction of a possible relapse of GD [14].

The presence of TgAb in GD is even less well studied. It was associated mainly with the prevalence and pathophysiology of GO [17, 19-21]. Most of those studies were of moderate size ( $<100$ patients), several studies were retrospective analyses. As those two antibodies are often measured in GD with or without GO, it is possible to link their positivity and levels to several clinical, ultrasound and hormonal parameters at the presentation of GD in an attempt to predict the future course of the AITD and the possible association of GO. As far as we know, there are no published analyses of the differences in the clinical presentation of GD depending on the number of positive antibodies in addition to TRAb.

The aim of the present study was to examine the clinical characteristics of patients with newly diagnosed GD who differ in the number of positive antithyroid antibodies as well as to find associations of the TPOAb and TgAb levels with important clinical, hormonal and ultrasound parameters.

\section{MATERIALS AND METHODS}

\section{Design}

This cross-sectional study included newly diagnosed GD patients from a tertiary hospital-based specialized endocrine unit. The study was approved by the Ethical committee of the Scientific Council at the Medical University Sofia (Research Project 13/2012, written consent) and was conducted in compliance with ethical standards and the Declaration of Helsinki. All patients signed informed consent prior to any procedure. All procedures were part of the routine clinical work-up in AITD. The inclusion criteria were age above 18 years, presence of newly diagnosed GD and willingness to participate. The exclusion criteria were glucocorticoid, interferon or any other type of immune-modulating treatment for concomitant disease.

\section{Methodology}

Complete physical examination was performed including eye symptoms, cardio-vascular signs of thyrotoxicosis and palpation of the thyroid. Smoking status, presence of concomitant autoimmune diseases and family history for thyroid disease were reviewed.

The serum levels of the thyroid hormones (TSH, free T4 and free T3) and antibodies (TRAb, TPOAb, $\mathrm{TgAb}$ ) were measured from a fasting morning blood sample with third generation ECLIA assays (Roche Diagnostics, Switzerland) on an Elecsys 2010 analyzer. The following reference ranges were applied: TSH (0.27-4.2 mU/l); fT4 (12-22 pmol/l); fT3 (3.54$6.47 \mathrm{pmol} / \mathrm{l}) ; \operatorname{TPOAb}(0-34 \mathrm{IU} / \mathrm{ml}) ; \operatorname{TgAb}(0-115 \mathrm{IU} /$ $\mathrm{ml})$; TRAb $(<1.75 \mathrm{IU} / \mathrm{l})$. Thyroid ultrasound was performed with a $9 \mathrm{MHz}$ linear transducer on a FukudaDenshi 550 device (Fukuda Corp., Japan). The thyroid volume was calculated in $\mathrm{ml}\left(\mathrm{cm}^{3}\right)$ according to the formula by Brunn et al. [22].

Thyroid Volume of the studied lobe $\left(\mathrm{cm}^{3}\right)=$ anteroposterior size $\mathrm{x}$ transverse size $\mathrm{x}$ length $\mathrm{x} 0.479$ (all in centimeters).

The presence of nodules was also reported. Additionally, power and color Doppler ultrasound was performed on a $14 \mathrm{MHz}$ Ultrasonix device (Ultrasonix Medical Corp., Burnaby, Canada) for vascularity and blood flow assessment. The diagnosis of GD was made in the presence of thyrotoxicosis (clinical or subclinical) with elevated levels of TRAb, diffuse hypoechogeneity of the thyroid parenchyma on ultrasound with increased blood flow on Doppler ultrasound [23]. Whenever available a technetium scan was reviewed and increased uniform uptake by the thyroid was used as confirmation [24]. Patients were evaluated by the clinical endocrinologists and experienced ophthalmologists for the presence of thyroidassociated ophtalmopathy (GO). The disease severity (according to the NOSPECS classification) and activity (according to the clinical activity score, CAS) were recorded [25].

Patients with elevated levels of TRAb only were marked as group 1+, those with an additional positive TPOAb or TgAb were marked as group $2+$, and those who were positive for all the three antibodies - as group 3+. Patients who had elevated levels of TPOAb were considered as TPOAb + and those with elevated levels of $\operatorname{TgAb}-\mathrm{as} \operatorname{TgAb}+$.

\section{Statistical analysis}

Statistical analyses were done using the SPSS 23.0 statistical package for Windows (SPSS Inc., Chi- 
cago, IL, USA). Descriptive statistics and variation analysis were first performed. A normal distribution was tested by the Shapiro-Wilk and KolmogorovSmirnov tests. Descriptive statistics were compared between groups via the Mann-Whitney and CruscalWallis tests for continuous variables. ANOVA and correlation analyses were performed. Missing values were not included in the computations. Statistical significance was set as $p \leq 0.05$; all tests were two-tailed.

\section{RESULTS}

249 patients with newly diagnosed GD (191 women, 58 men) agreed to participate. Their mean age was $44.2 \pm 14.4$ years (women only $-44.2 \pm 14.6$ years; men only $-44.0 \pm 14.0$ years). $40.2 \%$ of them (39.2\% of women and $48.2 \%$ of men) had symptoms and signs of accompanying GO. The baseline characteristics of the study population are presented in Table 1. There were no significant differences between men and women in their baseline characteristics. $28.7 \%$ of all participants were current smokers ( $29.1 \%$ of women and $27.5 \%$ of men), $18.7 \%$ were previous smokers $(17.3 \%$ of women and $23.5 \%$ of men) and $52.6 \%$ were never smokers $(53.6 \%$ of women and $49.0 \%$ of men). In $30.2 \%$ there was family history for thyroid disease was positive (32.8 $\%$ of women and $21.2 \%$ of men). $8.5 \%$ of patients had a concomitant autoimmune disease ( $2 \%$ vitiligo, $2 \%$ type 1 diabetes mellitus, $4.5 \%$ - other autoimmune disorder).

$64 \%$ of all patients were TPOAb positive and $36 \%$ were $\mathrm{TgAb}$ positive. $51 \%$ of all patients had enlarged thyroids (using cutoffs of $>18.0 \mathrm{ml}$ for females and $>25.0 \mathrm{ml}$ for males). $40 \%$ of the patients had symptoms and/or signs of GO. It was active in $71 \%$ of them. $21 \%$ of the patients had thyroid nodules on ultrasound.
Patient characteristics according to the number of positive antibodies

The patients were equally distributed according to the number of positive antibodies. In one third TRAb was the only elevated antibody, one third had additionally elevated TPOAb or TgAb (in $11 \%$ of them it was TgAb and in 89\% - TPOAb. In the remaining one third all three antibodies were elevated (Figure 1).

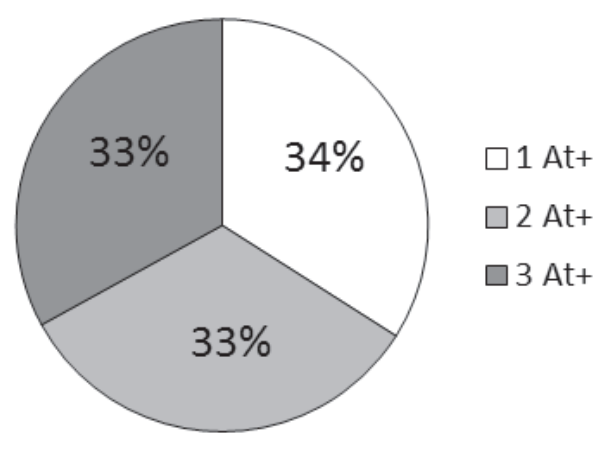

Fig. 1. The distribution of the patients according to the number of positive thyroid antibodies is shown

The patients' characteristics according to the number of positive antibodies are displayed as medians in Figure 2. Patients with more positive antibodies tended to be younger (Figure $2 \mathrm{~A}$ ). The median fT4 was lower in patients who were only TRAb positive (Figure 2 B). The fT3 levels did not differ among the groups. Patients with more positive antibodies had higher levels of TRAb (Figure $2 \mathrm{C}$ ), TPOAb (Figure $2 \mathrm{D}$ ) and $\operatorname{TgAb}$ (Figure $2 \mathrm{E}$ ). The thyroid volume was rising with the accumulation of positive antibodies (Figure $2 \mathrm{~F}$ ) and the prevalence of goiter on US was highest in the $3+$ group $(61 \%)$ and lowest in TRAb only positive patients (Figure $2 \mathrm{G}$ ). Most of the patients with enlarged thyroid volume were in group 3+ (40\%), while those TRAb only positive constituted

Table 1. The baseline characteristics of the participants are shown as median values and range

\begin{tabular}{|l|c|c|c|c|c|c|}
\hline & \multicolumn{2}{|c|}{ All subjects (N = 249) } & \multicolumn{2}{c|}{ Women (N=191) } & \multicolumn{2}{c|}{ Men (N = 58) } \\
\hline & Median & Range & Median & Range & Median & Range \\
\hline Cigarettes/d (if current smoker) & 12.5 & $5-40$ & 10.0 & $5-40$ & 17.5 & $5-20$ \\
\hline TSH, mUl/L & 0.005 & $0.001-0.410$ & 0.005 & $0.001-0.169$ & 0.005 & $0.001-0.410$ \\
\hline fT4, pmol/L & 45.5 & $14.1-145.6$ & 45.3 & $14.1-45.5$ & 45.5 & $15.5-100.0$ \\
\hline fT3, pmol/L & 13.2 & $4.2-50.1$ & 13.2 & $4.3-50.0$ & 13.2 & $4.2-50.1$ \\
\hline TRAb, UI/L & 9.0 & $1.9-40.00$ & 8.7 & $2.1-40.0$ & 11.6 & $1.9-40.0$ \\
\hline TPOAb, Ul/ml & 91.2 & $5.0-2199.0$ & 75.8 & $5.0-2199.0$ & 147.2 & $5.0-1000.0$ \\
\hline TgAb, Ul/ml & 50.5 & $10.0-4441.0$ & 42.1 & $10.0-4000.0$ & 80.0 & $10.0-4441.0$ \\
\hline Thyroid volume, $\mathrm{ml}$ & 18.5 & $5.0-112.1$ & 17.0 & $5.0-112.1$ & 22.0 & $9.0-77.0$ \\
\hline
\end{tabular}


$27 \%$ of the subgroup with goiter. The presence of thyroid nodules was negatively correlated with the number of positive antibodies (Figure $2 \mathrm{H}$ ). Nodules were seen more often in TRAb only positive patients. $63 \%$ of all nodules were found in this subgroup, while $20 \%$ of the nodules were found in group $2+$ and $17 \%$ - in group 3+. The presence of GO was negatively associated with the number of positive antibodies (Figure $2 \mathrm{I}$ ). Almost $2 / 3$ of patients in group $1+$ had GO vs. less than $1 / 3$ of the patients in the other two groups. $51 \%$ of the patients with accompanying GO were in the TRAb only group (1+), while $24 \%$ and $25 \%$ of them were in group $2+$ and $3+$ respectively. The severity of GO didn't differ among the groups.

Patient characteristics according to the presence of TPOAb (see Figure 3)

$64 \%$ of the patients were TPOAb+ $(n=160)$ with a median TPOAb level of $232.9 \mathrm{lU} / \mathrm{ml}$ (range 40.0-
$2199.0 \mathrm{IU} / \mathrm{ml})$. TPOAb levels were negatively correlated with the patients' age $(r=0.182, p=0.004$, $)$ and those who were TPOAb+ were younger (40 vs. 46.5 years, $p=0.004$; Figure $3 A$ ). TPOAb+ patients had higher fT 4 levels -46.2 vs. $36.3 \mathrm{mU} / \mathrm{l}(p=0.018$, Figure $3 \mathrm{~B}$ ). There was no correlation between the TPOAb levels and $\mathrm{TT} 3$. TPOAb levels were positively correlated with the TgAb (linear $r=0.614, p<0.005$ ) and TRAb levels $(r=0.204, p=0.003)$. TPOAb+ patients had higher TgAb and TRAb levels (Figure $3 \mathrm{C}$ and $3 \mathrm{D}$ ). Furthermore, the highest levels of each thyroid antibody were observed in the group where all three antibodies were positive $(p<0.005)$.

The thyroid volume was positively correlated with TPOAb (linear $r=0.193, p=0.004$ ). TPOAb+ patients had higher thyroid volumes than TPOAb- (Figure 3 E). Patients with increased thyroid volume had higher TPOAb levels - $219.3 \mathrm{IU} / \mathrm{ml}$ versus $173.6 \mathrm{IU} / \mathrm{ml}$ in

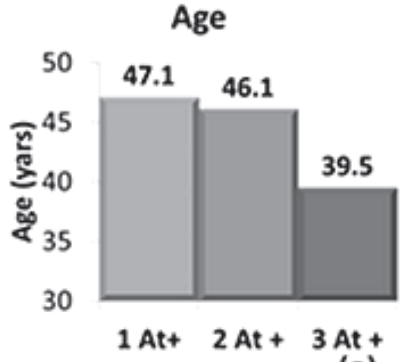

(a)

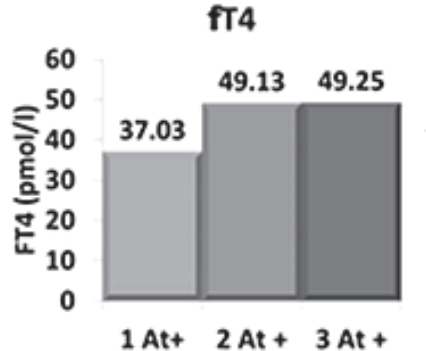

(b)

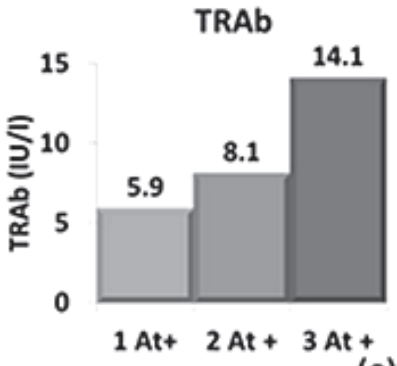

(c)

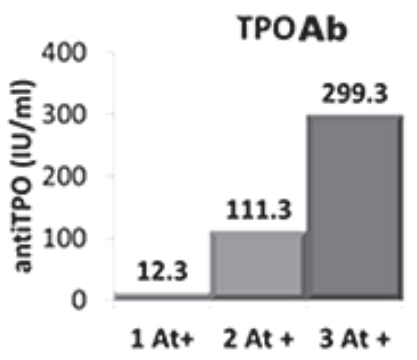

(d)

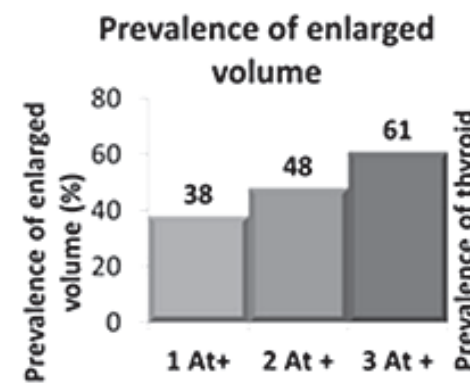

(g)

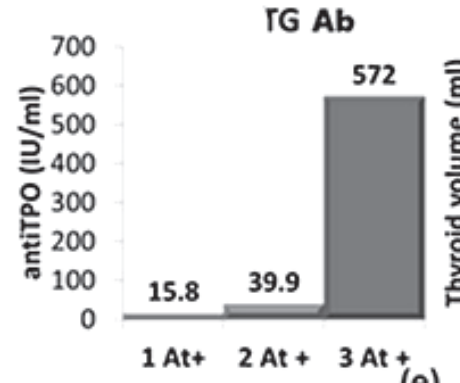

(e)
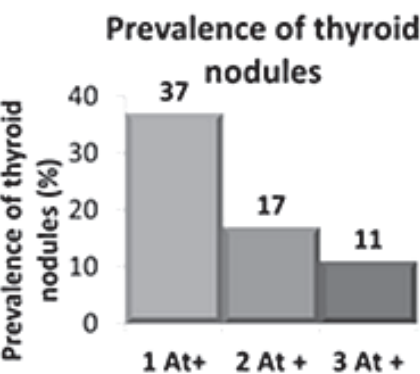

0

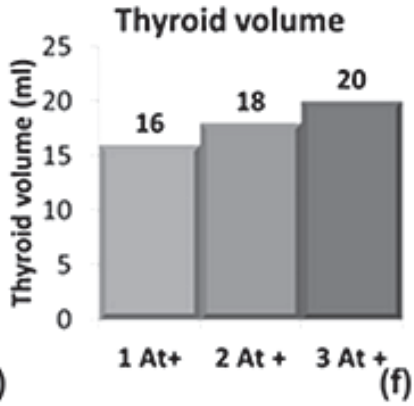

Prevalence of $\mathbf{G O}$

57

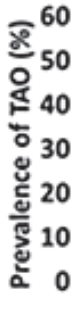

(h)

(A) mean age $(p<0.005)$; (B) median fT4 ( $p=0.014)$; (C) median TRAb ( $p=0.018)$; (D) median TPOAb $(p<0.005)$; (E) median TgAb $(p<0.005) ;(F)$ median thyroid volume $(p=0.015) ;(G)$ prevalence of goiter on US $(p=0.013) ;(H)$ prevalence of thyroid nodules on US $(p<0.005)$; (I) prevalence of $\mathrm{GO}(\mathrm{p}=0.002)$

Fig. 2. The distribution of patients with different number of positive antibodies is shown below according to their main characteristics (the significance of inter-group differences is shown in brackets) 
those with normal volumes, respectively $(p=0.046)$. $73 \%$ of the patients with goiter on US were TPOAb+, while only $56 \%$ of those with normal volume were TPOAb+ $(p=0.011)$ (Figure $3 F)$.

The prevalence of thyroid nodules was lower in TPOAb+ patients (Figure $3 \mathrm{G}, \mathrm{p}<0.001$ ) and it was negatively correlated with TPOAb levels $(p<0.005$, $r=0.233$ ). Only $35.5 \%$ of patients with nodules were TPOAb+, while in those without nodules $71.4 \%$ were aniTPO+.

The prevalence of $\mathrm{GO}$ was lower in TPOAb+ patients (Figure $3 \mathrm{H}, \mathrm{p}=0.004$ ) and its severity showed a negative association with the TPOAb levels $(r=$ $0.165, p=0.009$ ). Half of the patients with $G O$ were TPOAb+ $(53.5 \%)$, while in those without GO TPOAb were positive in $70.8 \%$. There was no association between the disease severity and activity and the TPOAb presence or levels.

Patient characteristics according to the presence of TgAb

TgAb were positive in $36.4 \%$ of all GD patients (in $34.4 \%$ of the women and in $43.1 \%$ of men). Regression analyses (curve estimation procedure) showed correlations of $\mathrm{TgAb}$ levels with $\mathrm{fT} 4$ (quadratic model, $R 2=0.733, p=0.019$ ), TPOAb levels (linear model, $\mathrm{R} 2=0.987, p<0.001$ ), the thyroid volume in $\mathrm{ml}$ (linear model, $R 2=0.698, p=0.005$ ), the severity of $G O$ (quadratic model, R2 $=0.657, p=0.041$ ) and the number of cigarettes per day in smokers (linear model, R2 $=0.836, p=0.005)$. TgAb titers were not correlated with TSH, $\mathrm{TT} 3$, levels of TRAb or activity of GO.

There were significant differences in the clinical characteristics between $\mathrm{TgAb}$ positive and $\mathrm{TgAb}$ negative patients. TgAb positive patients were younger (42.3 versus 46.0 years, $p<0.001)$, had lower TSH $(0.027$ versus $0.089 \mathrm{mUI} / \mathrm{l}, \mathrm{p}=0.006)$ and $\mathrm{fT} 4$ levels $(56.7$ versus $58.8 \mathrm{pmol} / \mathrm{l}, \mathrm{p}<0.001)$. In addition they had higher levels of TPOAb (281.4 versus $28.48 \mathrm{Ul} / \mathrm{mL}$, $\mathrm{p}<0.001)$, TRAb (12.82 vs. $7.21 \mathrm{Ul} / \mathrm{mL}, \mathrm{p}<0.001)$, thyroid volume (19 versus $17 \mathrm{ml}, \mathrm{p}<0.001$ ).

\section{DISCUSSION}

In this cross-sectional study we examined the prevalence and clinical correlations of TPOAb and TgAb positivity in Graves' disease. We found that approximately $2 / 3$ of the patients had positive TPOAb, while TgAb were positive in approximately $1 / 3$ only. The antibody positivity followed a pattern of thirds: one $1 / 3$ of the patients were only TRAb-positive, while $1 / 3$ had two positive antibodies (the one being invariably TRAb), and $1 / 3$ - all three positive antibodies. Positive TPOAb antibodies were found in younger patients, with higher fT4 and TRAb levels, higher thyroid volume and lower prevalence of nodules and GO. The positive TgAb were also found in patients at younger age, higher thyroid volumes, fT4, TRAb and TPOAb levels, as well as with lower TSH levels and severity of GO. The same trend was seen if these clinical parameters were correlated with the number of positive antibodies.

Elevated levels of TPOAb and TgAb are hallmarks of the underlying autoimmune disease and are more often screened for in autoimmune thyroiditis. The prevalence of positive TPOAb and TgAb antibodies in the setting of GD has been studied in the last 20 years. However, advances in immunology have modified the laboratory techniques for antibody detection and refined the search for different epitopes [13, 26-28]. This might affect the prevalence of antibody positivity as well as their predictive power. After all, very little is known about the associations of TPOAb and TgAbpositivity and different characteristics of GD.
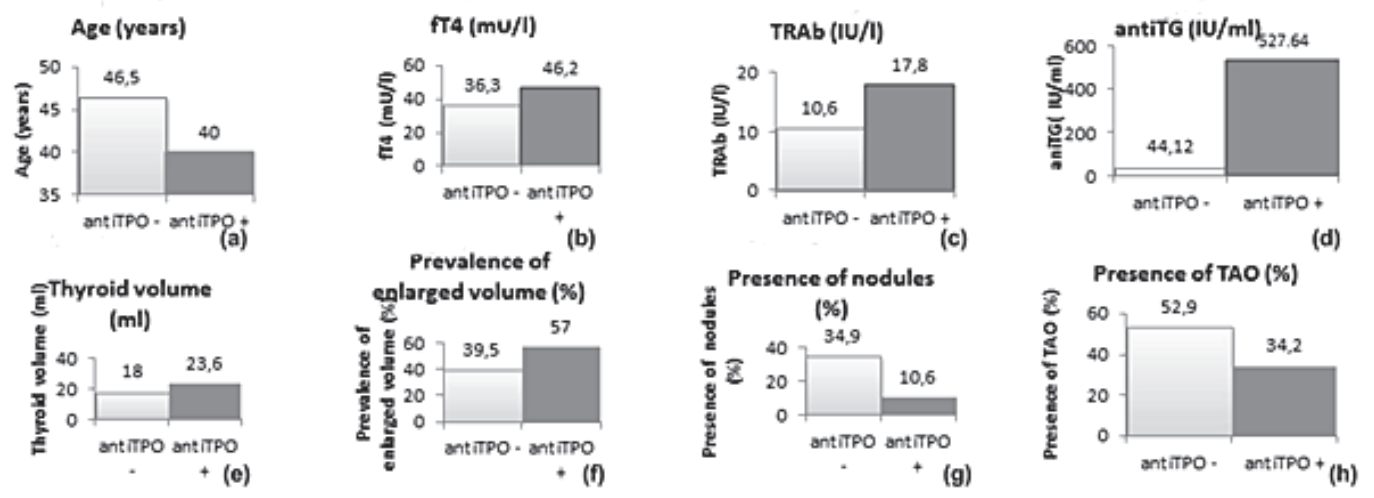

(A) patients' age $(p=0.004)$; (B) fT4 levels ( $p=0.018)$; (C) TRAb levels $(p=0.001 /$; (D) TgAb levels $(p=0.001)$; (E) thyroid volume $(p=0.01)$; $(F)$ prevalence of enlarged volume $(p=0.01) ;(G)$ presence of thyroid nodules $(p<0.001)$; and $(H)$ presence of $G O(p=0.004)$

Fig. 3. The differences in the hormonal and ultrasound patients' characteristics according to the TPOAb positivity or negativity are shown (the significance of inter-group differences is shown in brackets) 
Publications around the millenium have reported different numbers for the prevalence of TPOAb and TgAb positivity. S. Goh et al. compared thyroid antibody profiles in patients referred to Thyroid Clinics and Eye Clinics: TPOAb was positive in $79.6 \%$ and $43.7 \%$, while $\mathrm{TgAb}$ - in $73.2 \%$ and $36.7 \%$ respectively [8]. D. Khoo et al. found 91.2\% TPOAb positivity in GD and $62.8 \%$ - in GO, while TgAb positivity was $77.2 \%$ and $65.1 \%$ [9]. Both author groups suggested higher risks for GO in TRAb only positive patients. Testing new quantitative measurements of autoantibodies J. Gilmour et al. registered 87\% TPOAb and $73 \%$ TgAb positivity in typical GD patients [4]. In a national study TPOAb were positive in $86.3 \%$ and $\mathrm{TgAb}-$ in $43.1 \%$ of all GD patients [7]. All these studies were of moderate size (with a mean of around 100 participants). More recent studies have focused mainly on TPOAb positivity, which was found in $76.4 \%$ of 802 French patients with GD 15; in $7 \%$ of 111 Omani GD patients [11] and in $56.8 \%$ of 44 patients with subclinical hyperthyroidism due to GD [29]. Our data of $64 \%$ TPOAb-positivity and 36\% $\mathrm{TgAb}$ positivity show a lower prevalence than those in the listed publications. The proportions of patients with two or three positive thyroid antibodies were not reported separately in the literature. Y. Hu et al. studied 49 GD patients and found all three positive antibodies in $48.9 \%$ which is higher than our result of only $33 \%$ [30].

A more important question is whether finding positive TPOAb and/or TgAb in GD can add practical information for the clinician. GD patients may be stratified according to the number of positive antibodies at disease presentation. Those groups would differ in age, levels of FT4 and TRAb, thyroid volume and the presence of $\mathrm{GO}$ or thyroid nodules. Younger age of patients with higher numbers of positive antibodies would suggest a more complex immune intolerance. Levels of fT4 and thyroid volume could affect the dose and schedule of antithyroid drug treatment. The presence of nodules is crucial for performing FNA and screening for possible thyroid malignancies, which are more often encountered in nodules in the setting of GD [31, 32]. The two most important predictions would concern the possible future manifestation of $\mathrm{GO}$ as well as the probability for remission of the GD itself.

Considered separately TPOAb positivity in our newly diagnosed GD patients was, associated with higher thyroid volume, fT4 levels and lower prevalence of $\mathrm{GO}$ and thyroid nodules. Two author groups found that absent TPOAb antibodies and high TRAb levels identified a group at markedly increased risk of ophtalmopathy [8, 33, 34]. In a previous work we came to a similar conclusion: TPOAb positive GD patients rarely developed $\mathrm{GO}$, while $\mathrm{TgAb}$ positivity was associated with an increased risk [17]. A recent study in $399 \mathrm{GD}$ patients confirmed the protective role of TPOAb positivity in relation to $G O$ [12]. In this study TPOAb $<20 \mathrm{kIU} / \mathrm{L}$ and/or TRAb $>6.3 \mathrm{IE} / \mathrm{L}$ at the time of GD diagnosis were associated with an increased risk of developing GO after the diagnosis of GD was made [12]. Although the causative effect of TPOAb in GO might be questionable, there are studies detecting thyroid peroxidase mRNA and protein in the orbital tissue [35]. Our finding of a positive association of TPOAb levels with serum fT4 is intriguing, but it could be also due to a lower type II deiodinase enzyme activity [36]. In a study addressing the natural history of subclinical hyperthyroidism in GD, positive TPOAb were associated with risk of progression to overt hyperthyroidism - with hazard ratios from 1.06 to 10.15 per year [29]. In a histological study higher TPOAb levels were correlated with intra-thyroidal germinative center development, suggesting a more aggressive immunological attack [37]. The associations of TPOAb with higher thyroid volumes and a lower prevalence of nodules still need an explanation. We found practically no previous studies addressing these issues. A great contribution of TPOAb positivity would be the prediction of the probability for remission (or risk of relapse) after successful treatment with ATD. There are studies confirming improved prediction by using the combination of TRAb and TPOAb antibodies, especially when TRAb levels remain in the nondiagnostic "grey" zone [14]. Others found no use of TPOAb levels at GD presentation in predicting the risk of future relapses $[7,10]$. A few authors found non-significant trends for higher TPOAb in the presence of GD relapse [2, 38]. Anyhow, our study performed a cross-section at study presentation and is therefore not suited to answer the question about future relapses.

Considering $\operatorname{TgAb}$ positivity separately, our observations were not quite different from those about isolated TPOAb positivity. Both antibodies were positively correlated $(r=0.614 ; p<0.005)$. From a clinician's perspective $\operatorname{TgAb}$ are less prevalent and may be less useful than TPOAb for prediction of thyroid dysfunction and the disease course. The role of $\mathrm{TgAb}$ has been examined with special focus on GO. Thyroglobulin was identified in orbital tissues of patients with GO [39]. This is in line with the original hypothesis of J.P. Kriss et al. for an ethiological role of TgAb in GD presentation and progression [40], which was endorsed by other investigators, as well as recently reviewed [20]. Immunization with thyroglobulin induced Graves-like disease in mice [41]. Absent TgAb were associated with higher prevalence of $\mathrm{GO}$ in the hall- 
mark studies by S. Goh et al., and D. Khoo et al. [9, $33,34]$. In a previous study we came to the opposite conclusion - TgAb positivity was associated with an increased risk for the presence of GO [17]. Some authors reported a limited usefulness of anti-peroxidase and anti-thyrogobulin assays in GD patients and recommended testing for TPOAb without TgAb as a cost-effective approach [10]. Others found a place of $\mathrm{TgAb}$ assays in the diagnosis of GD only if TPOAb were negative [13]. TgAb might also be used for the prediction of future or expected relapses (respectively at disease presentation and at treatment end). The data on that topic are very scarce. A national study found that $\operatorname{TgAb}$ negativity was predictive for better outcomes (no relapse) both at GD presentation and treatment end [7]. Therefore, the place of $\mathrm{TgAb}$ in GD seems to need future clarification.

Our study has some limitations. First we weren't able to determine the biological effects of TRAb (stimulating, blocking or cleavage antibodies) and to assess if the type was relevant for the clinical presentation, especially the presence of $\mathrm{GO}$ as previously discussed [42]. Finally, our results might be affected by the fact that the study was performed in a specialized tertiary clinic, which might have introduced a bias towards more resistant or severe cases.

\section{CONCLUSIONS}

The different clinical characteristics of GD patients with different thyroid antibody profiles may be due to a variation in the pathogenesis of the disease itself. It seems appropriate to follow the progression of the disease, treatment response and complications in patients with different antibody profiles. A different approach based on the antibody profiles might be suitable.

\section{Ethical considerations}

Ethical clearance for this study was obtained from the Medical Ethics Committee of the Medical University of Sofia.

\section{Disclosure}

This study was partly supported by the $\mathrm{PhD}$ program 2016 at the Medical University Sofia.

\section{Conflicts of interest}

The authors declare no conflicts of interest.

\section{REFERENCES}

1. Ross DS, Burch HB, Cooper DS, et al. 2016 American Thyroid Association guidelines for diagnosis and management of hyperthyroidism and other causes of thyrotoxicosis. Thyroid 2016; 26: 1343-1421.
2. Tun NN, Beckett G, Zammitt NN, et al. Thyrotropin receptor antibody levels at diagnosis and after thionamide course predict Graves' Disease relapse. Thyroid 2016; 26: 1004-1009.

3. Sahlmann CO, Siefker U, Lehmann K, et al. Quantitative thyroid scintigraphy for the differentiation of Graves' disease and hyperthyroid autoimmune thyroiditis. Nuklearmedizin 2004; 43: 124-128.

4. Gilmour J, Brownlee Y, Foster P, et al. The quantitative measurement of autoantibodies to thyroglobulin and thyroid peroxidase by automated microparticle based immunoassays in Hashimoto's disease, Graves' disease and a follow-up study on postpartum thyroid disease. Clin Lab 2000; 46: 57-61.

5. McLachlan SM, Rapoport B. Why measure thyroglobulin autoantibodies rather than thyroid peroxidase autoantibodies? Thyroid 2004; 14: 510-520.

6. Ogawa T, Sakata S, Nakamura S, et al. Thyroid hormone autoantibodies in patients with Graves' disease: effect of antithyroid drug treatment. Clin Chim Acta 1994; 228: 113-122.

7. Gerenova JB, Vulkov Y, Boeva S, Halacheva K. Diagnostic and prognostic value of antithyroid antibodies and serum thyroglobulin levels during treatment of Graves' disease with Methimazole. J Endocrinologia 2001; 6: 16-18.

8. Goh SY, Ho SC, Seah LL, Fong KS, Khoo DH. Thyroid autoantibody profiles in ophthalmic dominant and thyroid dominant Graves' disease differ and suggest ophthalmopathy is a multiantigenic disease. Clin Endocrinol (Oxf) 2004; 60: 600-607.

9. Khoo DH, Ho SC, Seah LL, et al. The combination of absent thyroid peroxidase antibodies and high thyroid-stimulating immunoglobulin levels in Graves' disease identifies a group at markedly increased risk of ophthalmopathy. Thyroid 1999; 9: 1175-1180.

10. Wright-Pascoe R, Smikle MF, Barton EN, James OB. Limited usefulness of antithyroperoxidase and antithyroglobulin assays in Jamaicans with Graves' disease. Hum Antibodies 1999; 9: 161-164.

11. Alnaqdy A, Al-Maskari M. Determination of the levels of antithyroid-stimulating hormone receptor antibody with thyroid peroxidase antibody in Omani patients with Graves' disease. Med Princ Pract 2005; 14: 209-212.

12. Lantz M, Planck T, Asman P, Hallengren B. Increased TRAb and/or low anti-TPO titers at diagnosis of graves' disease are associated with an increased risk of developing ophthalmopathy after onset. Exp Clin Endocrinol Diabetes 2014; 122: 113-117.

13. Lastrzebska-Bohaterewicz E, Wojciechowska W, Gardas A. Place of thyroglobulin antibodies assay in laboratory diagnostic of autoimmune thyroid diseases. Endokrynol Pol 2005; 56: 30-34.

14. Schott M, Eckstein A, Willenberg HS, Nguyen TB, Morgenthaler NG, Scherbaum WA. Improved prediction of relapse of Graves' thyrotoxicosis by combined determination of TSH receptor and thyroperoxidase antibodies. Horm Metab Res 2007; 39: 56-61.

15. Goichot B, Bouée S, Castello-Bridoux C, Caron P. Survey of clinical practice patterns in the management of 992 hyperthyroid patients in France. Eur Thyroid J 2017; 6: 152-159.

16. Laurberg $P$, Nygaard B, Andersen $S$, et al. Association between TSH-receptor autoimmunity, hyperthyroidism, goitre, and orbitopathy in 208 patients included in the Remission Induction and Sustenance in Graves' Disease Study. J Thyroid Res 2014;2014:165487.

17. Boyanov M, Bakalov D, Sheinkova G. Levels of thyroid autoantibodies in patients with Graves Disease and Graves Ophtalmopathy. Turk J Endocrinol Metab 2010; 14: 50-53. 
18. Lee JH, Park SH, Koh DG, Suh BK. Thyroid peroxidase antibody positivity and triiodothyronine levels are associated with pediatric Graves' ophthalmopathy. World J Pediatr 2014; 10 : 155-159.

19. Komaki T, Sakata S, Nakamura S, et al. Fluctuations in the titers of anti-thyroid hormone and anti-thyroglobulin antibodies in 4 cases of Graves' disease during long-term treatment period. Nihon Naibunpi Gakkai Zasshi 1989; 65: 627-639.

20. Marinò $M$, Chiovato $L$, Lisi $S$, et al. Role of thyroglobulin in the pathogenesis of Graves' ophthalmopathy: the hypothesis of Kriss revisited. J Endocrinol Invest 2004; 27: 230-236.

21. Shanmuganathan $T$, Girgis $C$, Lahooti $H$, et al. Does autoimmunity against thyroglobulin play a role in the pathogenesis of Graves' ophthalmopathy: a review. Clin Ophthalmol 2015; 9: 2271-2276.

22. Brunn J, Block U, Ruf G, et al. Volumetrie der schilddrüse mittels Real-time-sonographie. Deutsche Medizinische Wochenschrift 1981; 106: 1338-1340.

23. Niedziela M, Warzywoda M, Korman E. Thyroid echogeneity as a useful tool for the differential diagnosis of hyperthyroidism in the course of Graves disease and Hashimoto thyroiditis. Endokrynol Diabetol Chor Przemiany Materii Wieku Rozw 2000; 6: 143-150.

24. Sahlmann CO, Siefker U, Lehmann K, et al. Quantitative thyroid scintigraphy for the differentiation of Graves' disease and hyperthyroid autoimmune thyroiditis. Nuklearmedizin 2004; 43: 124-128.

25. Bartalena L, Baldeschi L, Dickinson AJ, et al. Consensus statement of the European group on Graves' orbitopathy (EUGOGO) on management of Graves' orbitopathy. Thyroid 2008; 18: 333-346.

26. Chardès $T$, Chapal $N$, Bresson $D$, et al. The human anti-thyroid peroxidase autoantibody repertoire in Graves' and Hashimoto's autoimmune thyroid diseases. Immunogenetics 2002; 54: 141-157.

27. Gardas A, Lewartowska A, Sutton BJ, et al. Human thyroid peroxidase (TPO) isoforms, TPO-1 and TPO-2: analysis of protein expression in Graves' thyroid tissue. J Clin Endocrinol Metab 1997; 82: 3752-3757.

28. Bossowski A, Stasiak-Barmuta A, Czarnocka B, et al. Application of mouse monoclonal antibodies for identification of antigen regions of human thyroid peroxidase in adolescents with Graves' disease and non-toxic multinodular goiter by flow cytometry. Autoimmunity 2005; 38: 605-611.

29. Zhyzhneuskaya S, Addison C, Tsatlidis V, et al. The Natural history of subclinical hyperthyroidism in Graves' Disease: The rule of thirds. Thyroid 2016; 26: 765-769.
30. Hu YM, Liu W, Lu GH. Significance of combining tests of thyroid autoantibodies in differential diagnosis between Graves's disease and Hashimoto's hyperthyroidism. Zhonghua Yi Xue Za Zhi 2003; 83: 940-942.

31. Lima, PC, Moura Neto A, Tambascia MA, Zantut Wittmann $D E$. Risk factors associated with benign and malignant thyroid nodules in autoimmune thyroid diseases. ISRN Endocrinol 2013: 673146. DOI: 10.1155/2013/673146.

32. Scherer T, Wohlschlaeger-Krenn E, Bayerle-Eder M, et al. A case of simultaneous occurrence of Marine - Lenhart syndrome and a papillary thyroid microcarcinoma. BMC Endocr Disord 2013; 13: 16.

33. Goh SY, Ho SC, Seah LL, et al. Thyroid autoantibody profiles in ophthalmic dominant and thyroid dominant Graves' disease differ and suggest ophthalmopathy is a multiantigenic disease. Clin Endocrinol (Oxf) 2004; 60: 600-607.

34. Khoo DH, Eng PH, Ho SC, et al. Graves' ophthalmopathy in the absence of elevated free thyroxine and triiodothyronine levels: prevalence, natural history, and thyrotropin receptor antibody levels. Thyroid 2000; 10: 1093-1100.

35. Lai OF, Zaiden N, Goh SS, et al. Detection of thyroid peroxidase mRNA and protein in orbital tissue. Eur J Endocrinol 2006; 155: 213-218.

36. Molnár I, Szentmiklósi JA, Somogyiné-Vári É. Hyperthyroidism in patients with Graves' ophthalmopathy, and thyroidal, skeletal and eye muscle specific type 2 deiodinase enzyme activities. Exp Clin Endocrinol Diabetes 2017; 125: 514-521.

37. Segundo $\mathrm{C}$, Rodríguez $\mathrm{C}$, Aguilar $\mathrm{M}$, et al. Differences in thyroid-infiltrating B lymphocytes in patients with Graves' disease: relationship to autoantibody detection. Thyroid 2004; 14: 337-344.

38. Vos XG, Endert E, Zwinderman AH, et al. Predicting the risk of recurrence before the start of antithyroid drug therapy in patients with Graves' hyperthyroidism. J Clin Endocrinol Metab 2016; 101: 1381-1389.

39. Marinò M, Lisi S, Pinchera A, et al. Identification of thyroglobulin in orbital tissues of patients with thyroid-associated ophthalmopathy. Thyroid 2001; 11: 177-185.

40. Konishi J, Herman MM, Kriss JP. Binding of thyroglobulin and thyroglobulin-antithyroglobulin immune complex to extraocular muscle membrane. Endocrinology 1974; 95: 434-446.

41. Endo T, Kobayashi T. Immunization with thyroglobulin induces Graves'-like disease in mice. J Endocrinol 2009; 202: 217-22.

42. Kampmann E, Diana T, Kanitz M, Hoppe D, Kahaly GJ. Thyroid stimulating but not blocking autoantibodies are highly prevalent in severe and active thyroid-associated orbitopathy: A prospective study. Int J Endocrinol 2015:678194. 